\title{
Potencial de impacção dos caninos superiores: estudo radiográfico
}

\author{
- Ana Paula de Almeida Gomes Centro Universitário de Volta Redonda (UniFOA), Volta Redonda, RJ, Brasil \\ - Camila Gobbi de Carvalho Barbosa Centro Universitário de Volta Redonda (UniFOA), Volta Redonda, RJ, Brasil \\ - Pedro Augusto Peixoto Bittencourt Centro Universitário de Volta Redonda (UniFOA), Volta Redonda, RJ, Brasil \\ - Roberta Mansur-Caetano Centro Universitário de Volta Redonda (UniFOA), Volta Redonda, RJ, Brasil
}

RESUMO | Objetivo: o objetivo deste artigo foi revisar a literatura sobre os preditores radiográficos e a extração do canino decíduo para interceptar a impacção do canino superior permanente, assim como fez parte do seu escopo avaliar uma amostra do fenômeno. Métodos: foi feita uma revisão da literatura e um estudo observacional utilizando-se os preditores radiográficos, no sentido horizontal e angular, em uma amostra de dez pacientes ortodônticos, com acompanhamento radiográfico de um caso. Resultados: a extração do canino superior decíduo com o objetivo de redirecionar o trajeto eruptivo do elemento sucessor é indicada quando o dente, pela radiografia panorâmica, localiza-se no setor 2 com angulação igual ou maior que $20^{\circ}$ a $30^{\circ}$; no setor 3 ; no setor 4 com angulação igual ou menor que $20^{\circ}$ a $30^{\circ}$. A amostra totalizou uma avaliação de 20 caninos superiores, dos quais 30\% apresentavam indicação da conduta interceptativa. Em um dos casos, o elemento 13 estava localizado no setor 2, no limite com o setor 3 , e com angulação de $29^{\circ}$. Portanto, foi realizada exodontia do elemento 53 e um ano depois pôde-se identificar radiograficamente o redirecionamento da trajetória eruptiva do elemento 13 . Conclusões: na presente amostra, 30\% dos caninos possuíam indicação de extração do antecessor decíduo, sendo que no caso acompanhado observou-se o benefício do procedimento interceptativo referente a impacção.

DESCRITORES | Dente Canino; Dente Impactado; Radiografia Panorâmica.

ABSTRACT | Maxillary canines impaction potential: a radiographic study • Objectives: Review the literature on radiographic predictors and extraction of the deciduous canine to intercept impaction of the upper permanent canine, and subsequently, evaluate a sample. Methods: Literature review and observational study using radiographic predictors, in horizontal and angular direction, in a sample of 10 orthodontic patients, with a radiographic follow-up of one case. Results: The extraction of primary canine with the objective of redirecting the eruptive path of the successor element is indicated when it is located, by panoramic radiography, in sector 2 with angulation equal to or greater than $20^{\circ}$ to $30^{\circ}$; in sector 3 ; in sector 4 with angulation equal to or less than $20^{\circ}$ to $30^{\circ}$. The sample comprised an evaluation of 20 maxillary canines, $30 \%$ of which had indication for the interceptive conduct. In one of the cases, element 13 was located in sector 2 (limit with sector 3 ) with a $29^{\circ}$ angulation, therefore, extraction of element 53 was performed and a year later the redirection of the eruptive path of element 13 could be identified radiographically. Conclusions: In the present sample, 30\% of the canines had an indication of extraction of the deciduous predecessor, and in the accompanied case, the benefit of the interceptive procedure regarding impaction was observed.

DESCRIPTORS | Canine Tooth; Tooth Impacted; Radiography Panoramic.

AUTOR CORRESPONDENTE $\mid \bullet$ Roberta Mansur Caetano Centro Universitário de Volta Redonda • Rua 40, 20, sala 418 Volta Redonda, RJ, Brasil • 27260-200 E-mail: robmansur@hotmail.com

- Recebido $23 \mathrm{Fev}, 2021$ • Aceito $07 \mathrm{Mar}, 2021$

- Dol http://dx.doi.org/10.11606/issn.2357-8041.clrd.2021.182404 


\section{INTRODUÇÃO}

Os caninos superiores permanentes apresentam o trajeto irruptivo mais extenso de toda a dentição, o que os tornam mais susceptíveis a ocorrência de impacções e retenções. ${ }^{1}$

A impacção ou impactação é caracterizada quando o dente não erupciona na arcada no período esperado; para os caninos, a média é de 13 anos nos meninos e de 12 anos e 3 meses nas meninas. Dessa forma, a detecção de anormalidades deve acontecer em torno de 8 anos para que a melhor conduta preventiva seja realizada. ${ }^{2,3}$

Para um diagnóstico correto quanto ao potencial de impacção dos caninos superiores, é importante que a anamnese seja feita de forma criteriosa, analisando os antecedentes familiares, além da realização do exame físico seguido do exame radiográfico em idade oportuna. ${ }^{4}$

A radiografia panorâmica está indicada no diagnóstico e no acompanhamento do tratamento do canino potencialmente impactado, ${ }^{5}$ pois permite a visualização da angulação, da altura do posicionamento do canino e de sua distância ao plano sagital mediano de acordo com a sobreposição aos incisivos adjacentes. ${ }^{6}$

$\mathrm{Na}$ avaliação do posicionamento intraósseo dos caninos superiores pela radiografia panorâmica, utiliza-se três parâmetros radiográficos: no sentido horizontal, vertical e angular.7-10 Porém, Naoumova e Kjellberg ${ }^{9}$ afirmam que as avaliações no sentido horizontal e angular já são bons preditores radiográficos de impacção.

De acordo com Ericson e Kurol, ${ }^{11}$ a avaliação horizontal indica a posição da coroa do canino no sentido mésio-distal em relação aos incisivos lateral e central, sendo representado por setores que vão de 1 a 5. Já a avaliação angular é a análise realizada entre o eixo longo do canino e a linha média facial, representados pelo ângulo chamado alfa (Figura 1).7-10

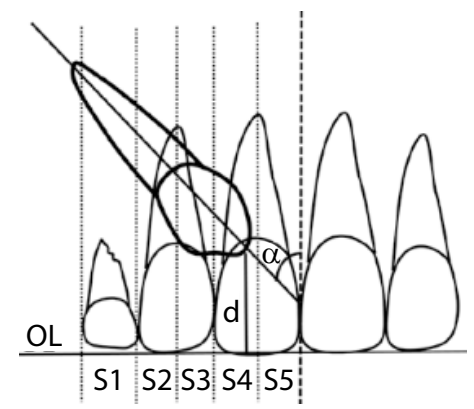

Figura 1 | Posicionamento dos caninos nos sentidos, horizontal (setores 1 a 5) e angular (ângulo alfa).

Fonte: Naoumova e Kjellberg. ${ }^{9}$

O diagnóstico precoce e o início do tratamento antecipado visam evitar que um canino potencialmente impactado se torne, de fato, um canino impactado. ${ }^{12}$ Existem diversas possibilidades de tratamento para esse fenômeno, dentre elas podese citar a extração do próprio canino permanente impactado, a extração do canino decíduo, a exposição cirúrgica para tracionamento ortodôntico e o transplante autógeno. ${ }^{4,13-15}$

A extração do canino permanente impactado é indicada em situações em que há reabsorção radicular externa ou interna, dilaceração da raiz, presença de anquilose, impactação grave, oclusão funcional satisfatória na qual o primeiro pré-molar ocupa o lugar do canino e a presença de alterações patológicas. ${ }^{16}$

Entretanto, o tratamento interceptativo realizado com a extração do canino decíduo é a forma ideal de tratamento, visto que atua na prevenção da impactação do canino superior, estimulando a erupção espontânea e corrigindo sua trajetória para que alcance um bom alinhamento no plano oclusal. Além disso, esse tratamento reduz as chances de reabsorção da raiz do incisivo lateral e evita a necessidade de procedimento cirúrgico para tracionamento ortodôntico, promovendo melhores resultados a longo prazo.7-9,11-12

O sucesso deste tipo de tratamento está diretamente relacionado à idade do paciente e à severidade da impactação. Alguns procedimentos 
interceptativos podem ser utilizados em associação à extração do canino decíduo: a prevenção da migração mesial fisiológica dos molares permanentes, o uso do arco transpalatino ou do aparelho extraoral para a distalização dos elementos e a expansão rápida da maxila. ${ }^{4,8,12}$

A impactação do canino superior pode gerar diversas complicações ao paciente tanto do ponto de vista funcional, pois pode resultar em desequilíbrio na oclusão, como estético, pois pode ocasionar desarmonia do sorriso. Além disso, a impactação pode causar reabsorção da raiz dos incisivos permanentes, anquilose e, em alguns casos, pode desenvolver cistos e aumentar o tempo de tratamento ortodôntico se este for iniciado de forma tardia., ${ }^{4,17-21}$

O objetivo deste estudo foi tanto revisar a literatura sobre os preditores radiográficos e a extração do canino decíduo como forma de interceptar a impacção do canino superior permanente como avaliar de amostra de pacientes das Clínicas de Ortodontia do Centro Universitário de Volta Redonda (UniFOA) que apresentavam essa condição.

\section{MÉTODOS E MATERIAIS}

Este estudo foi aprovado pelo Comitê de Ética em Pesquisa do Centro Universitário de Volta Redonda, em 18/11/2019, com número CAAE 24354919.8.00oo.5237.

Para a construção do referencial teórico, foram analisados trabalhos científicos, principalmente a partir de 2015, indexados nas bases de dados LILACS, MEDLINE e SciELO.

No estudo observacional da amostra, foram utilizados os preditores radiográficos no sentido horizontal e angular na avaliação das radiografias panorâmicas dos pacientes das Clínicas de Ortodontia do UniFOA que iniciaram tratamento de setembro de 2019 a março de 2020. Tal análise foi realizada por três avaliadores autores desse estudo, dois graduandos e um professor doutor na área de imaginologia e especialista em ortodontia responsável pela calibração, que identificaram a prevalência de casos com potencial de impacção e indicação de extração do canino decíduo.

Foram incluídos pacientes de ambos os gêneros, selecionados aleatoriamente, de 7 a 11 anos de idade, com presença dos incisivos laterais nos estágios 9 (raíz quase completa e ápice aberto) e 10 de Nolla (raíz completa e fechamento apical), ${ }^{22}$ com presença dos caninos superiores decíduos, unilateral ou bilateralmente e com caninos superiores em formação, não irrompidos. Foram excluídos os pacientes com transposição dentária que envolvesse o canino superior.

Das radiografias panorâmicas dos pacientes avaliados que iniciaram tratamento ortodôntico, dez se enquadravam nos critérios de inclusão desse estudo. Assim sendo, foi possível acompanhar um paciente com indicação de exodontia do canino decíduo superior, como conduta preventiva da impacção do respectivo canino permanente.

\section{RESULTADOS}

$\mathrm{Na}$ análise do posicionamento intraósseo dos 20 caninos superiores pela radiografia panorâmica, foram utilizados dois preditores radiográficos referentes a impactação no sentido horizontal (setores) e angular.

No sentido horizontal, foram identificados $60 \%$ dos elementos (12) no setor 1, sendo que 58,33\% (7) eram do lado direito e 41,66 \% (5) do lado esquerdo; $25 \%$ (5) no setor 2, sendo 40\% (2) do lado direito e 60\% (3) do lado esquerdo; $10 \%$ (2) no setor 3 , sendo $50 \%$ (1) do lado direito e 50\% (1) do lado esquerdo; e 5\% (1) no setor 4, localizado no lado esquerdo. Já no sentido angular, $75 \%$ dos caninos (15) possuíam angulação de até $19^{\circ}$ e $25 \%(5)$, de $20^{\circ}$ a $30^{\circ}$.

Analisando os resultados citados acima, 30\% (6) do total de caninos avaliados possuíam indicação para extração do antecessor decíduo, sendo que 
50\% (3) localizavam-se no setor 2 e apresentavam as seguintes angulações: $21^{\circ}, 23,5^{\circ}$ e $29^{\circ} ; 33,33 \%$ (2) estavam localizados no setor 3 , com angulações de $9^{\circ}, 13,5^{\circ}$; e $16,66 \%$ (1) no setor 4 , com angulação de $21^{\circ}$. Dessa forma, os pacientes foram encaminhados para exodontia dos elementos decíduos.

No caso acompanhado pelos professores do curso de pós-graduação em ortodontia, a paciente foi diagnosticada com mordida cruzada anterior dento alveolar e tratada com expansão rápida da maxila e protração maxilar. Ao final do tratamento, com 7 anos e 11 meses de idade, foi detectado um significativo potencial de impacção do elemento 13, localizado no setor 2 (limite com setor 3 ) e angulação de $29^{\circ}$. Foi realizada exodontia do elemento $53 \mathrm{e}$, um ano depois, pode-se identificar o redirecionamento da trajetória eruptiva do elemento 13 (Figuras 2 e 3).

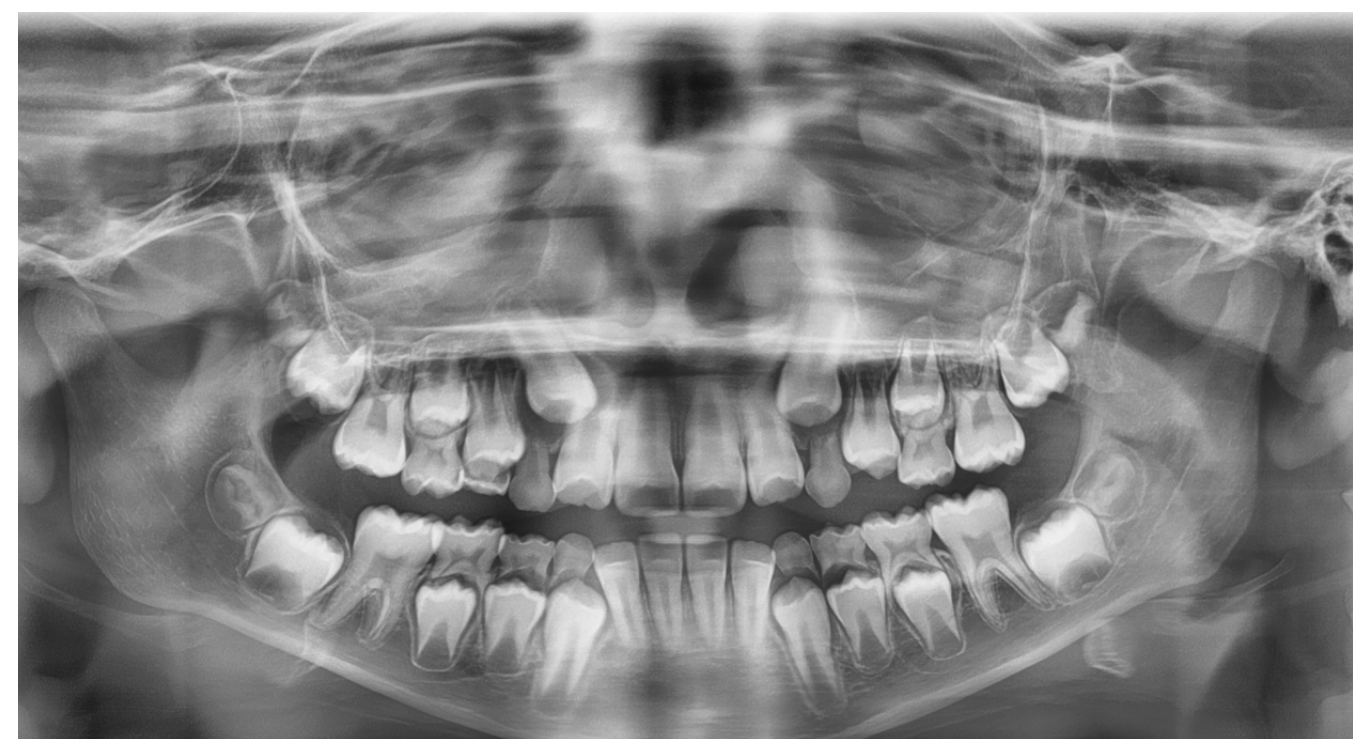

Figura 2 | 7 anos e 11 meses de idade - elemento 13 no setor 2 (limite com setor 3) e angulação de $29^{\circ}$.

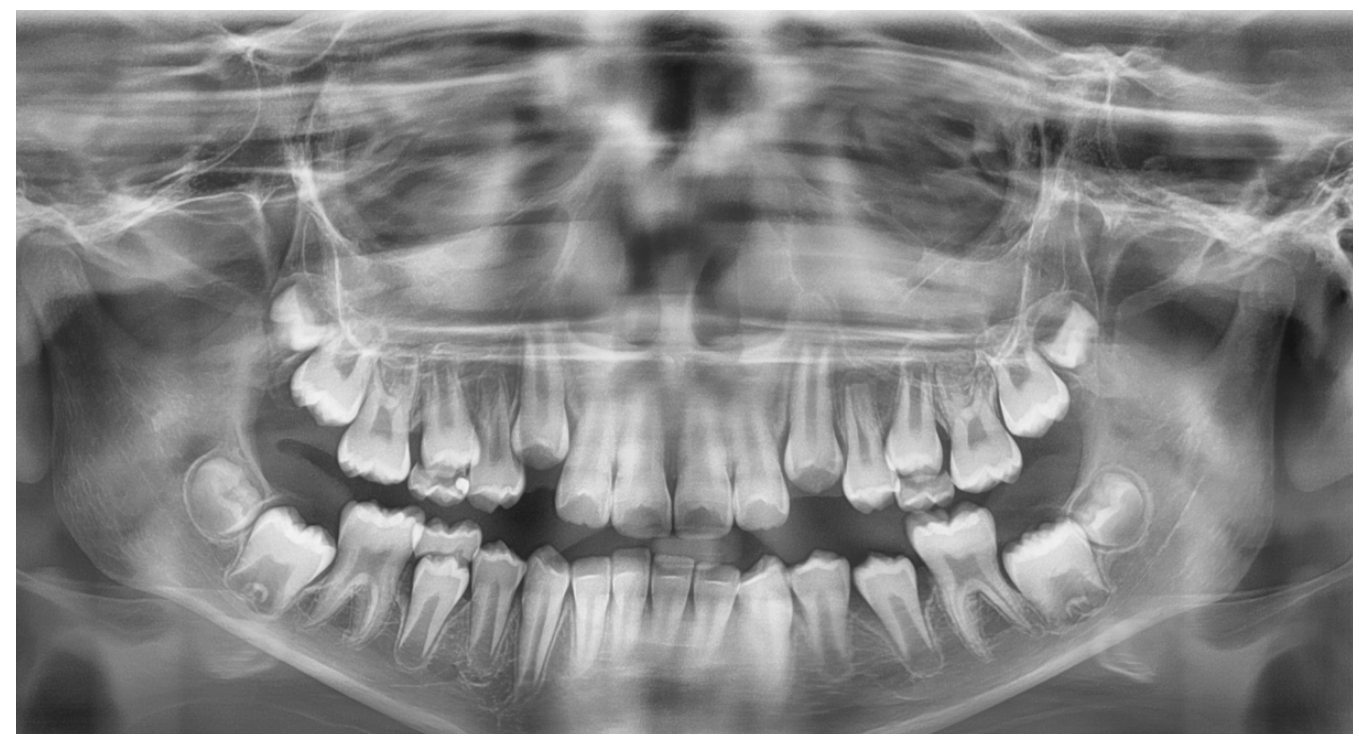

Figura 3 || 8 anos e 11 meses de idade - redirecionamento da trajetória eruptiva do elemento 13. 


\section{DISCUSSÃO}

De acordo com os preditores radiográficos, diversos estudos indicam a extração do canino superior decíduo quando o respectivo canino permanente se localiza no setor 2 com angulação igual ou maior que $20^{\circ}$ a $30^{\circ}$; no setor 3 , independente de sua angulação; e no setor 4 com angulação igual ou menor que $20^{\circ}$ a $30^{\circ}$. Entretanto, se estiver no setor 4 e com angulação maior que $30^{\circ}$, deve-se realizar imediato procedimento cirúrgico para tracionamento ortodôntico ou extração. ${ }^{7-11}$

O prognóstico do tratamento interceptativo é desfavorável quando a coroa do canino está mais próxima da linha média facial do paciente, como nos setores 4 e 5), quando o elemento está mais alto que o plano oclusal ou quando apresenta angulação maior que $30^{\circ}$ em relação à linha média. Outros fatores que podem influenciar no prognóstico são a idade do paciente e o desenvolvimento radicular. ${ }^{7-9}$

No presente estudo, 30\% dos caninos superiores avaliados tinham indicação de extração do canino decíduo. No caso acompanhado, a paciente com idade aproximada de 8 anos apresentava o elemento 13 no setor 2 com angulação de $29^{\circ}$ e, portanto, possuía prognóstico favorável.

Após a extração do canino decíduo, o monitoramento da irrupção do canino permanente deve ser feito com análise clínica e radiográfica com um intervalo de 6 meses a 18 meses..$^{11}$ Entretanto, se após esse período não houver melhora no posicionamento do canino permanente, recomendase a exposição cirúrgica para tracionamento ortodôntico ou a extração. ${ }^{23}$

$\mathrm{O}$ estudo de Ericson e Kurol ${ }^{11}$ demonstrou excelente resultado dessa forma de tratamento interceptativo. Ao avaliarem 46 caninos permanentes, $78 \%$ erupcionaram normalmente após a extração do canino decíduo, com um intervalo de tempo entre 6 e 12 meses.

Outros estudos também comprovaram a utilização dessa abordagem precoce e conservadora para interceptar a irrupção ectópica dos caninos superiores, tais como Power e Short, ${ }^{24}$ com $62 \%$ de taxa de sucesso; Baccetti, Leonardi e Armi, ${ }^{25}$ em que 92 caninos superiores foram estudados com taxa de erupção espontânea após a extração dos caninos decíduos de 65,2\%; e as revisões sistemáticas feitas por Almasoud ${ }^{26}$ e por Alyammahi, Kaklamanos, e Athanasiou; ${ }^{27}$ além de diversos estudos. ${ }^{10,28-30}$

Com o caso acompanhado, foi possível demonstrar a eficácia desse tratamento. Foi realizado a extração apenas do canino decíduo, o que permitiu o redirecionamento irruptivo do elemento sucessor permanente, corroborando com o estudo de HadlerOlsen et al., ${ }^{31}$ no qual foi comparado o impacto da extração do canino decíduo com a extração do canino do $1^{0}$ molar decíduos em 48 casos de caninos superiores permanentes potencialmente impactados, chegandose à conclusão de que o resultado foi semelhante em ambos os grupos. Portanto, recomenda-se o procedimento interceptativo por ser menos invasivo com a extração de apenas um elemento decíduo.

A associação da extração do canino decíduo com técnicas expansionistas na arcada superior aumentam as taxas de sucesso da intervenção precoce, ${ }^{1,8}$ como visto no caso citado.

\section{CONCLUSÃO}

Na amostra avaliada, 30\% dos caninos possuíam indicação de extração do antecessor decíduo para minimizar o potencial de impacção. No caso acompanhado, foi observado o benefício desse procedimento interceptativo em relação à impacção.

\section{REFERÊNCIAS}

1. Garib DG, Aiello CA. Distúrbios irruptivos dos caninos superiores permanentes. In: Silva Filho OG, Garib DG, Lara TS. Ortodontia Interceptiva: protocolo de tratamento em duas fases. São Paulo: Artes Médicas; 2013. p. 303-33.

2. Kumar S, Mehrotra P, Bhagchandani J, Singh A, Garg A. Localization of Impacted Canines. J Clin Diagn Res, 2015; 9(1):11-4. doi: 10.786o/JCDR/2015/10529.5480. 
3. Elangovan B, Sathynarayana HP, Padmanabhan S. Effectiveness of various interceptive treatments on palatally displaced canine-a systematic review. Int Orthod. 2019;17(4):634-42. doi: 10.1016/j.ortho.2019.08.002.

4. Soares VK, Menezes LM. Abordagem de caninos superiores impactados. Rev Ortodon Gauch. 2016;20(1):4-12.

5. Carvalho PL, Lopes AMS, Silva CM, Assis ACS, Medeiros JMF. Utilização de protocolo radiográfico na clínica de Odontopediatria. Rev Bras Odontol. 2010;67(2):279-82. doi: 10.18363/rbo.v67n2.p.279.

6. Cappellette M, Cappellette M Jr, Fernandes LCM, Oliveira AP, Yamamoto LH, Shido FT, Oliveira WC. Caninos permanentes retidos por palatino: diagnóstico e terapêutica - uma sugestão técnica de tratamento. R Dental Press Ortodon Ortop Facial. 2008;13(1):60-73. doi: 10.1590/S1415-54192008000100008.

7. Alqerban A, Storms AS, Voet M, Fieuws S, Willems G. Early prediction of maxillary canine impaction. Dentomaxillofac Radiol. 2016;45(3):20150232. doi: 10.1259/dmfr.20150232.

8. Hoffelder LB. Efeitos da expansão rápida da maxila sobre o posicionamento dos caninos superiores potencialmente impactados na fase da dentadura mista [tese]. Porto Alegre: Universidade Federal do Rio Grande do Sul; 2016.

9. Naoumova J, Kjellberg $\mathrm{H}$. The use of panoramic radiographs to decide when interceptive extraction is beneficial in children with palatally displaced canines based on a randomized clinical trial. Eur J Orthod. 2018;40(6):565-74. doi: 10.1093/ ejo/cjyoo2.

10. Arriola-Guillén LE, Ruíz-Mora GA, Rodríguez-Cárdenas YA, Castillo AAD, Boessio-Vizzotto M, Silveira HLD. Influence of impacted maxillary canine orthodontic traction complexity on root resorption of incisors: A retrospective longitudinal study. Am J Orthod Dentofacial Orthop. 2019;155(1):28-39. doi: 10.1016/j.ajodo.2018.02.011.

11. Ericson S, Kurol J. Early treatment of palatally erupting maxillary canines by extraction of the primary canines. Eur J Orthod. 1988;10(4):283-95. doi: 0.1093/ejo/10.4.283.

12. Ferreira ES, Barros SEC, Chiqueto KFG, Hoffelder LB. Diagnóstico e alternativas de tratamento precoce para os caninos superiores potencialmente ectópicos. In: Schroeder MA, Artese F, Mattos C, Almeida R, editores. Conceitos, inovações e decisões na ortodontia. Proceedings of the 12th Congresso Internacional da Associação Brasileira de Ortodontia; 2019 Oct 9-12; Rio de Janeiro, Brazil. São José dos Pinhais: Plena; 2019. p. 61-73.

13. Giglio FPM, Gurgel JA. Abordagem cirúrgico ortodôntica de dentes não irrompidos. Ortodontia. 2010;43(2):169-75.
14. Simão TM, Neves MJG, Yamate EM, Crepaldi MV, Burger RC. Tracionamento ortodôntico de caninos superiores impactados por palatino. Rev FAIPE. 2012;2(1):29-40.

15. Kaczor-Urbanowicz K, Zadurska M, Czochrowska E. Impacted Teeth: An Interdisciplinary Perspective. Adv Clin Exp Med. 2016;25(3):575-85. doi: 10.17219/acem/37451.

16. Bishara SE. Impacted maxillary canines: a review. Am J Orthod Dentofacial Orthop. 1992;101(2):159-71. doi: 10.1016/0889-5406(92)70008-X.

17. Silva Filho OG, Fugio N, Capelozza Filho L, Cavassan AO. Irrupção ectópica dos caninos permanentes superiores: soluções terapêuticas. Ortodontia. 1994;27(1):50-66.

18. McSherry PF. The ectopic maxillary canine: a review. Br J Orthod. 1998;25(3):209-16. doi: 10.1093/ortho/25-3.209.

19. Cunha CI, Poletto CAR, Ignácio SA, Guariza Filho O, Tanaka O, Camargo ES. Associação entre caninos inclusos e agenesias de incisivos laterais superiores permanentes. Arch Oral Res. 2011;7(2):147-55. doi: 10.7213/aor.v7i2.23114.

20. Yan B, Sun Z, Fields H, Wang L. Maxillary canine impaction increases root resorption risk of adjacent teeth: a problem of physical proximity. Am J Orthod Dentofacial Orthop. 2012;142(6):750-7. doi: 10.1016/j.ajodo.2012.07.016.

21. Becker A, Chaushu S. Etiology of maxillary canine impaction: A review. Am J Orthod Dentofacial Orthop. 2015;58(1):55765. doi: 10.1016/j.ajodo.2015.06.013.

22. Nolla CM. The development of the permanent teeth. J Dent Child. 1960;27(4):254-66.

23. Oliveira GS, Brito HHA, Rezende H, Oliveira DD. Diagnóstico precoce e interceptação dos caninos superiores permanentes com direção ectópica de erupção. Rev Clin Ortodon Dental Press. 2010;8(6):57-60.

24. Power SM, Short MB. An investigation into the response of palatally displaced canines to the removal of deciduous canines and an assessment of factors contributing to favourable eruption. Br J Orthod. 1993;20(3):215-23. doi: 10.1179/bjo.20.3.215.

25. Baccetti T, Leonardi M, Armi P. A randomized clinical study of two interceptive approaches to palatally displaced canines. Eur J Orthod. 2008;30(4):381-5. doi: 10.1093/ejo/cjno23.

26. Almasoud NN. Extraction of primary canines for interceptive orthodontic treatment of palatally displaced permanent canines: A systematic review. Angle Orthod. 2017;87(6):87885. doi: 10.2319/021417-105.1.

27. Alyammahi AS, Kaklamanos EG, Athanasiou AE. Effectiveness of extraction of primary canines for interceptive management of palatally displaced permanent canines: a systematic 
review and meta-analysis. Eur J Orthod. 2017;40(2):149-56. doi: 10.1093/ejo/cjxo42.

28. Bedoya MM, Park JH. A review of the diagnosis and management of impacted maxillary canines. J Am Dent Assoc. 2009;140(12):1485-93. doi: 10.14219/jada.archive.2009.0099.

29. Litsas G, Acar A. A review of early displaced maxillary canines: etiology, diagnosis and interceptive treatment. Open Dent J. 2011;5:39-47. doi: 10.2174/1874210601105010039.
30. Benson PE, Graham EA, Parkin NA. Management of the palatally displaced canine. Clin Dent Rev. 2018;2:26. doi: 10.1007/ s41894-018-0039-z.

31. Hadler-Olsen S, Sjögren A, Steinnes J, Dubland M, Bolstad NL, Pirttiniemi P, et al. Double vs single primary tooth extraction in interceptive treatment of palatally displaced canines: A randomized controlled trial. Angle Orthod. 2020;90(6):751-7. doi: 10.1093/ejo/cjxo42. 